\title{
Manifestations buccales de la maladie de Behçet. A propos de 12 cas au Sénégal
}

\author{
Oral manifestations of Behcet's disease. 12 cases report in Senegal
}

PAUL NIANG ${ }^{1}$, SOUKEYE DIA TINE ${ }^{1}$, ABDOU BA $^{1}$, RUDOLPH DIOP 2 , BABACAR TAMBA ${ }^{1}$, BARRY GASSAMA ${ }^{1}$, CATHERINE BINTA ${ }^{1}$, SUZANE OUMOU NIANG ${ }^{3}$, BOUBACAR DIALLO ${ }^{1}$

\begin{abstract}
RÉSUMÉ
La maladie de Behçet est une affection rare, systémique, d'étiologie inconnue et d'allure polymorphe.

L'objectif de cette étude est de décrire les aspects épidémiologiques, cliniques et thérapeutiques de la maladie de Behçet au Sénégal mais aussi de préciser le rôle de l'odontologiste dans le diagnostic et la prise en charge de cette affection.

Les dossiers de patients ont été sélectionnés selon les critères diagnostiques du Groupe International d'Etude de la Maladie de Behçet, dans le service de Dermatologie du CHU Aristide Le Dantec de Dakar, entre décembre 1997 et mars 2006.

12 cas (8 hommes, 4 femmes) de maladie de Behçet, chez des sujets de race noire, âgés de 18 à 54 ans, ont été colligés. Les aphtes buccaux, sans caractères particuliers, étaient constants ; ils réalisaient une aphtose bipolaire dans 11 cas. Ces lésions buccales pouvaient avoir un caractère inaugural $(n=2)$ et contribuer à motiver la consultation $(n=10)$. Les principales manifestations systémiques étaient oculaires $(n=8)$, articulaires et cutanées ( $n=6$ pour chacune des localisations).

La maladie de Behçet chez les patients sénégalais est classique : elle se caractérise par la présence constante des aphtes buccaux, souvent associés à des aphtes génitaux (aphtose bipolaire) et à une uvéite antérieure. (Med Buccale Chir Buccale 2009 ; 15: 183-188).
\end{abstract}

mots clés: maladie de Behçet, aphtes buccaux récurrents

\section{$\underline{\text { SUMMARY }}$}

The aim of this study was to assess the importance of oral manifestations of Behcet's disease in a population of Senegalese patients.

We retrospectively studied epidemiologic aspects and clinical spectrum of 12 patients with Behcet's disease, fulfilling International Study Group for Behcet's Disease (ISGBD) criteria. This study was conducted in dermatologic unit clinic of the Aristide Le Dantec teaching hospital, during 9 years. We recorded the clinical features and compared the data with other recent statistics.

\footnotetext{
1. Service de Chirurgie buccale Département d'Odonto-Stomatologie Faculté de Médecine Pharmacie et Odonto-Stomatologie Dakar

2. Service de Stomatologie CHU Aristide Le Dantec Dakar

3. Service de Dermatologie CHU Aristide Le Dantec Dakar
}

Demande de tirés à part:

Paul Débé Amadou Niang Service de Chirurgie buccale Département d’Odonto-Stomatologie Faculté de Médecine Pharmacie et

Odonto-Stomatologie Université Cheikh Anta Diop BP 16162 Dakar Fann dbniang@hotmail.com 
All the patients were black (18- 54 years). The sex ratio was 2 (8 men, 4 women). The most frequent manifestations at disease onset were oral contributing to justify the consultation $(n=10)$, followed by genital aphthae $(n=9)$. The commonest manifestations observed throughout the disease course were oral aphthae $(n=12)$, genital aphthae $(n=11)$, uveitis $(n=8)$, various cutaneous lesions $(n=6)$ and arthritis $(n=6)$. Recurrent oral aphtae without typical physical characteristic carried out bipolar (oral and genital) aphtosis $(n=11)$. These oral lesions were inaugural $(n=2)$.

Behcet's disease in Senegalese patients is characterized by a variety of clinical manifestations in agreement with the medical literature. Oral manifestations are the major clinical signs, the next in common occurrence are genital and ocular lesions. (Med Buccale Chir Buccale 2009 ; 15: 183-188).

key words: Behçet's disease, recurrent aphtous stomatitis

médecine

buccale chirurgie buccale

VOL. $15, \mathrm{~N}^{\circ} 4$ 2009

page 184
La maladie de Behçet est une aphtose buccale ou, le plus souvent bucco-génitale, associée à des manifestations systémiques diverses dont les plus importantes sont oculaires, cutanées, nerveuses et vasculaires. Elle a comme substratum anatomique une vascularite [1].

Au Sénégal, les aphtes buccaux représentent des lésions fréquentes mais la maladie de Behçet est rare et méconnue. Les études menées sur cette affection en Afrique subsaharienne sont peu nombreuses et aucune ne semble avoir été effectuée par un odontologiste. Une série de 17 cas sénégalais a déjà été publié ${ }^{[2]}$, et un cas chez un Sénégalais vivant en France a également été rapporté ${ }^{[3]}$.

La maladie de Behçet peut mettre en jeu le pronostic fonctionnel (risque de cécité) et le pronostic vital lors de certaines atteintes viscérales $[4,5]$. L'objectif de cette étude était de décrire les aspects épidémiologiques, cliniques et thérapeutiques de la maladie de Behçet au Sénégal mais aussi de préciser le rôle de l'odontologiste dans le diagnostic et la prise en charge de cette affection.

\section{MATÉRIEL ET MÉTHODE}

II s'agit d'une étude rétrospective descriptive qui a été menée dans le service de Dermatologie du CHU Aristide Le Dantec de Dakar. Elle a concerné les dossiers des patients atteints de la maladie sur une période de 100 mois (de décembre 1997 à mars 2006).

Tous les cas de maladie de Behçet diagnostiqués selon les critères diagnostiques du Groupe International d'Etude de la Maladie de Behçet éta- blis en 1990 ont été recensés à partir des dossiers de consultation.

Ces critères sont des ulcérations buccales et génitales récurrentes, des lésions oculaires à type d'uvéite ou de vascularite rétinienne, des lésions cutanées à type d'érythème noueux, de pseudo-folliculite, de lésions papulo-pustuleuses ou de nodules acnéiformes, et un test pathergique positif. Les ulcérations buccales associées à au moins 2 signes supplémentaires sont nécessaires au diagnostic.

Les aspects épidémiologiques, cliniques et thérapeutiques ont été étudiés chez chaque patient.

\section{RÉSULTATS}

\section{Aspects épidémiologiques}

12 cas de maladie de Behçet ont été recensés sur une période de 100 mois soit une fréquence de 1,44 cas par an. Ces 12 cas de maladie de Behçet représentent $0,4 \%$ des 3218 patients hospitalisés dans le service pendant la période d'étude. L'âge des patients est présenté dans le Tab. 1. 8 patients étaient de sexe masculin, 4 de sexe féminin soit, un sex ratio de 2 . Tous les patients étaient de race noire et vivaient au Sénégal.

\section{Aspects cliniques}

Les ulcérations douloureuses étaient le motif de consultation dans tous les cas (Tab. 2).

Le délai avant la $1^{\text {re }}$ consultation est précisé dans le Tab. 3. Les aphtes buccaux étaient présents dans tous les cas, 11 cas sur 12 dans le cadre d'une aphtose bipolaire. La notion de récurrence a été rapportée dans 10 cas et la fréquence des poussées estimée à 3 ou 4 fois par an. 
Les lésions buccales ont été inaugurales dans 2 cas ; elles ont alors précédé de 2 ans les autres manifestations. Les aphtes buccaux étaient mineurs et multiples dans tous les cas. Leur topographie était variable : sur la langue dans 11 cas, la face interne des joues dans 3 cas, les lèvres dans 3 cas et le plancher dans 1 cas.

Tableau 1 : Nombre de cas en fonction de l'âge des patients.

Number of cases according to the age of the patients.

\begin{tabular}{|c|c|}
\hline AGE DES PATIENTS (ans) & Nombres de cas \\
\hline 18 & 2 \\
\hline 22 & 1 \\
\hline 25 & 2 \\
\hline 27 & 2 \\
\hline 28 & 1 \\
\hline 32 & 1 \\
\hline 38 & 1 \\
\hline 40 & 1 \\
\hline 54 & \\
\hline
\end{tabular}

Tableau 3 : Nombre de cas en fonction du délai de consultation.

Number of cases according to the waiting period before the consultation.

\begin{tabular}{|c|c|}
\hline $\begin{array}{c}\text { DELAIS } \\
\text { DE CONSULTATION }\end{array}$ & $\begin{array}{c}\text { Nombres } \\
\text { de cas }\end{array}$ \\
\hline 1 mois & 1 \\
\hline 2 mois & 1 \\
\hline 4 mois & 1 \\
\hline 6 mois & 3 \\
\hline 1 an & 1 \\
\hline 2 ans & 2 \\
\hline 3 ans & 1 \\
\hline 4 ans & 1 \\
\hline 6 ans & 1 \\
\hline
\end{tabular}

Les différentes manifestations systémiques associées sont répertoriées dans le Tab. 4. Les signes cutanés étaient à type de pseudofolliculite dans 3 cas et d'érythème noueux dans 3 cas. Les signes articulaires étaient à type de polyarthralgies d'allure inflammatoire dans 6 cas. Une uvéite a été confirmée par un examen ophtalmologique dans 8 cas. Une hyperthermie a été notée dans 5 cas. Le test pathergique, réalisé dans 6 cas, a été positif dans 3 cas.

Tableau 2 : Nombre de cas en fonction du motif de consultation.

Number of cases according to the purpose of the consultation.

\begin{tabular}{|c|c|}
\hline $\begin{array}{c}\text { MOTIFS DE } \\
\text { CONSULTATION }\end{array}$ & $\begin{array}{c}\text { Nombres } \\
\text { de cas }\end{array}$ \\
\hline Ulcérations buccales & 1 \\
\hline Ulcérations buccales et génitales & 6 \\
\hline Ulcérations buccales et cutanées & 2 \\
\hline $\begin{array}{c}\text { Ulcérations buccales, } \\
\text { génitales et cutanées }\end{array}$ & 1 \\
\hline Ulcérations génitales & 2 \\
\hline
\end{tabular}

médecine buccale chirurgie buccale VOL. $15, \mathrm{~N}^{\circ} 4$ 2009 page 185
Tableau 4 : Nombre de cas en fonction des manifestations systémiques.

Number of cases according to the systemic manifestations.

\begin{tabular}{c|c|}
\hline $\begin{array}{c}\text { MANIFESTATIONS } \\
\text { SYSTEMIQUES }\end{array}$ & $\begin{array}{c}\text { Nombres } \\
\text { de cas }\end{array}$ \\
\hline Signes articulaires & 6 \\
\hline Signes cutanés & 6 \\
\hline Signes digestifs & 2 \\
\hline Signes neurologiques & 5 \\
\hline Signes oculaires & 8 \\
\hline Signes cardiaques & 2 \\
\hline Signes ORL & 1 \\
\hline Signes pulmonaires & 1 \\
\hline Signes généraux & 5 \\
\hline
\end{tabular}




\section{Aspects thérapeutiques}

Seule la polyvidone iodée a été employée en bains de bouche pour le traitement local des aphtes buccaux. L'association corticoïdes-colchicine a été prescrite de façon systématique. Le thalidomide a été utilisé dans 2 cas (un cas d'aphte buccal réfractaire et un cas d'aphte génital géant). L'évolution de la maladie de Behçet a été favorable après une durée d'hospitalisation moyenne de 1 mois. Le nombre de récidives n'a pas pu être précisé.

\section{COMMENTAIRES}

12 cas de maladie de Behçet observés sur une période de 100 mois dans le service de Dermatologie du CHU de Dakar sont rapportés. Ces cas présentent des caractères classiques: fréquence faible, survenue à un âge jeune, pré- médecine buccale chirurgie buccale

VOL. $15, \mathrm{~N}^{\circ} 4$ 2009

page 186 dominance du sexe masculin, fréquence élevée de l'aphose bipôlaire (buccale et génitale) et de l'uvéite antérieure. La maladie de Behçet semble rare si l'on tient compte de sa fréquence annuelle dans le service (1,44 cas/an) qui, toutefois, est supérieure à celle rapportée dans une étude multicentrique réalisée au Sénégal entre 1970 et 1995 n'incluant aucun de ces cas (0,7 cas/an) [2] .

Cette affection est cosmopolite $[6,7]$. Sa prévalence varie selon les régions géographiques ${ }^{[7-9]}$; elle est faible en dehors des pays de la route de la soie, c'est-à-dire ceux de la région méditerranéenne orientale et de l'Asie centrale et orientale [2,5,10]. Les données concernant les Africains en zone tropicale sont quasi inexistentes ; la maladie n'a pas été décrite chez les Afro-américains et reste exceptionnelle chez les Subsahariens vivant en Europe ${ }^{[3]}$. Cependant, cette fréquence annuelle hospitalière est probablement inférieure à celle qui pourrait être observée dans la population globale en raison des patients échappant à la consultation, du recrutement dans d'autres services et des cas mineurs passant inaperçus. En outre, les critères diagnostiques peuvent pécher par défaut, ne permettant pas d'identifier un certain nombre de malades atteints d'une maladie de Behçet ${ }^{[1]}$. Cet inconvénient est aggravé par le fait que cette affection a une expression clinique et une base immunogénétique variable selon les pays et les ethnies ${ }^{[1,11]}$.
C'est une affection de l'adulte jeune, l'âge moyen étant de 29,5 ans. Ces résultats sont confirmés par d'autres études. Ainsi, l'âge de survenue est situé entre 20 et 30 ans ${ }^{[2,8,10]}$ et l'âge moyen entre 26 et 30,3 ans [2,5,12]. Et la maladie est rare avant la puberté ou après 50 ans ${ }^{[6,13,14]}$. L'atteinte prédominante des sujets de sexe masculin qui étaient au nombre de 8 (contre 4 de sexe féminin) s'inscrit dans les données de la littérature [2,8,10]. Cependant, l'androtropisme n'a été démontré que chez des sujets d'origine turque dans une étude menée en Allemagne ${ }^{[5]}$.

Le délai de consultation après l'apparition de la maladie varie de 2 mois à 6 ans avec une moyenne de 19 mois qui correspond au délai mentionné dans la plupart des études. Le délai moyen entre l'apparition des premiers signes et le diagnostic varie entre 36 et 54 mois ${ }^{[5,15]}$.

Les aphtes buccaux sont retrouvés dans les antécédents et/ou observés à l'inspection étaient notés dans tous les cas ; ils étaient associés à des aphtes génitaux réalisant une aphtose bipolaire dans 11 cas sur 12 . Ces résultats sont confirmés par la plupart des études qui montent que les aphtes buccaux constituent la manifestation la plus fréquente : ils sont présents dans 90 à $100 \%$ des cas ${ }^{[16-18]}$. Les manifestations cutanéomuqueuses observées depuis le début et à tous les stades de la maladie sont dominées par l'aphtose bipôlaire [18].

Dans cette série, aucun malade n'a été référé par un odontologiste alors que les aphtes buccaux sont fréquents et constituent des signes majeurs et souvent précurseurs de la maladie. Ceci reflète de la méconnaissance de cette affection en odontologie au Sénégal et contribue sans doute à ce que les formes frustres restent ignorées. L'aspect banal, la fréquence des poussées au moins triannuelle (10 cas), le caractère inaugural ( 2 cas), le nombre et le siège ubiquitaire sont en adéquation avec les résultats de nombreuses études. En effet, l'absence de caractères physiques particuliers des lésions buccales est démontré $[19,20]$. Et leur caractère inaugural est observé dans 25 à $86 \%$ des cas ${ }^{[4,5,8]}$. Cependant, ces lésions habituellement multiples peuvent être uniques ${ }^{[4]}$; les localisations sur le palais, le dos de la langue et l'oropharynx qui n'ont pas été observées dans 
nos cas, seraient statistiquement plus fréquentes dans la maladie de Behçet [4]. L'absence de notion de récurrence dans 2 cas pourrait s'expliquer par la précocité de la consultation à 2 et 6 mois après les premiers signes.

Diverses manifestations systémiques ont été observées : la plus fréquente était l'uvéite antérieure (8 cas), confirmée par un examen ophtalmologique. Ce polymorphisme clinique est largement corroboré avec des manifestations cutanées, oculaires, mais également neurologiques, vasculaires, gastro-intestinales et articulaires qui interpellent de nombreuses spécialités [5,7,19]. Parmi ces manifestations, une série confirme également la prédominance de l'uvéite au Sénégal [2]. Le test pathergique n'était pas systématique ; il a été réalisé dans 6 cas et positif dans 3 cas. La réaction pathergique n'est pas pathognomonique de la maladie de Behçet bien que sa spécificité semble élevée avec une positivité qui atteint $60 \%$ en zone d'endémie [5].

Si l'aphtose buccale est le pilier du diagnostic de la maladie de Behçet, la présence d'aphtes buccaux isolés peut rendre le diagnostic difficile, en absence de caractères particuliers. Les critères diagnostiques peuvent également pécher par excès favorisant la confusion avec plusieurs autres affections [1]. D'ailleurs, l'aphtose buccale récurrente et l'aphtose bipolaire peuvent être considérées comme des formes frustes de la maladie de Behçet : elles imposent la recherche de signes suggestifs de cette affection (pseudofolliculite, pathergie positive, uvéite antérieure, atteinte neurologique ou articulaire, phlébite superficielle, terrain génétique HLA-B5) et nécessitent, même en absence de tout autre signe, une surveillance régulière, dans l'éventualité de son développement [21-23].

La polyvidone iodée en bains de bouche a été systématiquement employée pour le traitement local des aphtes buccaux. Selon la littérature, les applications topiques sont les plus utilisées pour traiter les aphtes en nombre limité siégeant dans des aires faciles d'accès ${ }^{[5,18]}$. Par contre, la polyvidone iodée est sans effet sur la douleur et n'est indiquée que pour le contrôle de la contamination et de la surinfection [18,22,24]. Le recours fréquent à ce produit est probablement favorisé par sa large diffusion car il appartient à la liste des médicaments génériques essentiels établie lors de l'initiative de Bamako. Cette dernière définit une stratégie pour le renforcement des soins de santé primaire ; elle a été proposée en 1987 par l'OMS et adoptée par l'ensemble des pays de la région sahélienne. Malheureusement, en l'absence de consultation en odontologie, les patients ne bénéficient pas des mesures de promotion de l'hygiène bucco-dentaire, de l'élimination des facteurs locaux directs et des conseils d'alimentation inhérents au traitement local des aphtes buccaux ${ }^{[5,18,24]}$.

Les corticostéroïdes, les AINS et la colchicine font partie des traitements conventionnels [13,20,25]. La colchicine a longtemps été considérée comme le traitement de choix de la maladie de Behçet en raison de son efficacité sur presque toutes les manifestations ${ }^{[5,18,26]}$; pour certains auteurs, elle auraient peu d'effet sur les aphtes buccaux [18]. Les associations sont les plus efficaces en général ${ }^{[12]}$.

Les immunosuppresseurs dont le thalidomide font partie des traitements récents [20,26]. Le thalidomide qui est une alternative indiquée dans les formes réfractaires aux traitements conventionnels et notamment à l'association colchicine-dapsone, est efficace sur les aphtes buccaux sévères ou réfractaires [16,18,22].

Sur le plan évolutif, les agents thérapeutiques systémiques ont des effets équivoques dont l'évaluation est d'autant plus difficile que l'évolution de la maladie est imprévisible [12].

\section{CONCLUSION}

Même si nous ne pouvons établir une comparaison avec des études de grande envergure réalisées pour la plupart en zone d'endémie, nos observations correspondent aux données de la littérature.

La maladie de Behçet touche une population jeune et active, à prédominance masculine ; elle revêt un intérêt en odontologie, étant donné la présence quasi constante des aphtes buccaux. Les odontologistes peuvent jouer un rôle important dans le dépistage précoce de cette affection médecine buccale chirurgie buccale VOL. $15, \mathrm{~N}^{\circ} 4$ 2009 page 187 
en raison de ses lésions buccales qui motivent souvent la consultation et, parfois, de leur caractère inaugural.

Le suivi et le traitement local des lésions buccales semblent relégués au second plan s'il n'y a pas de consultation en odontologie. La prise en charge des patients atteints de la maladie de Behçet doit être pluridisciplinaire.

\section{RÉFÉRENCES}

1 - Hamza M. Maladie de Behçet (pp. 883-924) In : Kahn MF, Peltier AP, Meyer O, Piette JC ed. Les maladies et syndromes. Flammarion Médecine, Paris, 2000.

2 - Dia D, Dieng MT, Sy Ndiaye T, Fall S, Ndongo S, Diallo M, Moreira Diop T, Labou A, Ndiaye B. Behcet's disease in Dakar (Senegal): epidemiological and clinical features. Dakar Med 2003 ; 48 : 64-7.

3 - Simon F, Chouk PY, Aboumediene A, Maréchal H, Richalet S, Jeandel P. Maladie de Behçet chez le noir africain. Med Afrique Noire 1992 ; 39 : 658-64.

4 - Georgaklis CC. Behcet's disease and its oral manifestations. Dent Today $2004 ; 23: 92-5$.

médecine

buccale chirurgie buccale

VOL. $15, \mathrm{~N}^{\circ} 4$ 2009

page 188
5 - Altenburg A, Papoutsis N, Orawa H, Martus P, Krause L, Zouboulis CC. Epidemiology and clinical manifestations of Adamantiades-Behçet disease in Germany - Current pathogenetic concepts and therapeutic possibilities. J Dtsch Dermatol Ges 2006 ; 4 : 49-66.

6 - Al-Otaibi LM, Porter SR, Poate TW. Behcet's disease: a review. J Dent Res 2005 ; 84 : 209-22.

7 - Eguia A, Villarroel M, Martinez-Conde R, Echebarria MA, Aguirre JM. Adamantiades-Behcet disease: an enigmatic process with oral manifestations. Med Oral Patol Oral Cir Bucal 2006 ; 11 : E6-11.

8 - Bonfioli AA, Orefice F. Behcet's disease. Semin Ophthalmol $2005 ; 20$ : 199-206.

9 - Gul A. Behcet's disease as an autoinflammatory disorder. Curr Drug Targets Inflamm Allergy 2005 ; 4 : 81-3.

10 - Escudier M, Bagan J, Scully C. Number VII Behcet's disease (Adamantiades syndrome). Oral Dis 2006 ; 12 : 78-84.

11 - Tanaseanu S, Tanaseanu C, Pompilian V, Badea C. Clinical particularities in a Romanian series of Behcet's disease patients. Rom J Intern Med 2003 ; 41 : 41-51.

12 - Bang D. Clinical spectrum of Behcet's disease. J Dermatol $2001 ; 28: 610-3$.

13 - Al-Mutawa SA, Hegab SM. Behcet's disease. Clin Exp Med 2004 ; 4 : 103-31.
14 - Alpsoy E, Elpek GO, Yilmaz F, Ciftcioglu MA, Akman A Uzun S, Karakuzu A. Androgen receptor levels of oral and genital ulcers and skin pathergy test in patients with Behcet's disease. Dermatology 2005 ; 210 : 31-5.

15 - Lin J, Li H, Huang Y, Zheng K, Zhou Z, Lin X. Clinic analysis of Behcet disease. Yan Ke Xue Bao 2004 ; 20 : 155-7.

16 - Scully C. The mouth in dermatological disorders. Practitioner 2001 ; 245 : 942-4, 946, 949-52.

17 - Pipitone N, Boiardi L, Olivieri I, Cantini F, Salvi F, Malatesta R, La Corte R, Triolo G, Ferrante A, Filippini D, Paolazzi G, Sarzi-Puttini P, Restuccia G, Salvarani C. Clinical manifestations of Behcet's disease in 137 Italian patients: results of a multicenter study. Clin Exp Rheumatol 2004 ; 22 : S46-51.

18 - Alpsoy E. Behcet's disease: treatment of mucocutaneous lesions. Clin Exp Rheumatol 2005 ; 23 : 532-9.

19 - Faure M. Ulceration or erosion of the oral and (or) genital mucosa. Rev Prat $2002 ; 52$ : 649-55.

20 - Suzuki Kurokawa M, Suzuki N. Behcet's disease. Clin Exp Med 2004 ; 4 : 10-20.

21 - Boyvat A, Ekmekci P, Gurgey E. Bipolar aphthosis. A forme fruste of Behcet's disease. Long term followup of 26 cases. Adv Exp Med Biol 2003; $528: 321-2$.

22 - Mc Carty MA, Jorizzo JL. Complex aphthosis: evaluation for Behcet's disease? Adv Exp Med Biol 2003 ; $528: 303-10$.

23 - Yildirim M, Baysal V, Inaloz HS, Doguc D. The significance of serum nitric oxide levels in Behcet's disease and recurrent aphthous stomatitis. J Dermatol 2004 ; 31: 983-8.

24 - Bruce AJ, Rogers RS 3rd. Acute oral ulcers. Dermatol Clin $2003 ; 21: 1-15$.

25 - Evereklioglu C. Current concepts in the etiology and treatment of Behcet disease. Surv Ophthalmol 2005 ; $50: 297-350$.

26 - Sanchez-Negron FA. Behcet's disease. Dermatol Online J $2004 ; 10: 14$. 